\title{
Perceptual constancy of mechanical properties of cloth under variation of external forces
}

\author{
Wenyan $\mathrm{Bi}^{1}{ }^{*}$, Bei Xiao ${ }^{2}$ \\ ${ }^{1}$ Department of Psychology, American University, Washington, DC, USA \\ ${ }^{2}$ Department of Computer Science, American University, Washington, DC, USA
}
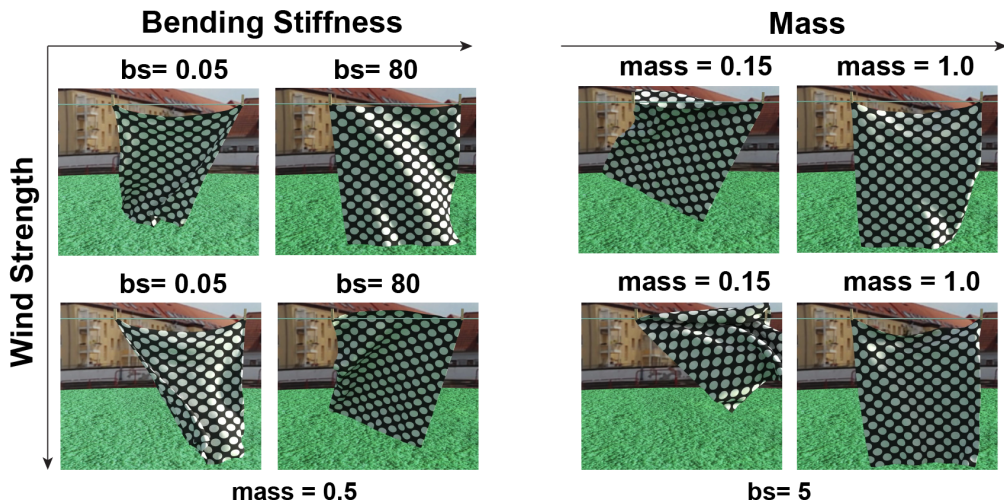

Figure 1: Dynamic information is important for perception of mechanical properties of cloth. Using this information, however, can be challenging because both the intrinsic mechanical properties and the external forces contribute to the apparent motion of fabrics. For example, lighter fabrics under weak wind can look similar to heavier fabrics under strong wind (the third image in the first row and the fourth image in second row). Can humans discount the external wind and have an invariant inference of the intrinsic mechanical properties of the cloth? If so, what visual cues allow them to achieve such constancy? We create animated videos where we we vary both the intrinsic mechanical properties (bending stiffness and mass) as well as the wind force, and measure the perception of mechanical properties of fabrics.

\section{Abstract}

Deformable objects such as cloth exhibit their mechanical properties (e.g. stiffness) through shape deformation over time under external forces. Mechanical properties are important because they tell us the affordance of the object and helps us predict what type of action can be done upon it. Previous research shows that motion statistics can be used to develop computer vision algorithms to estimate mechanical properties of cloth under an unknown wind force. It is unclear what motion cues human use to estimate mechanical properties. Estimating mechanical properties is difficult because both the intrinsic properties of the fabric and the external force contribute to the apparent motion of the fabric. However, in order to achieve invariant material perception, the visual system needs to discount the effects of external force. In this paper, we investigate whether humans have an invariant representation of mechanical properties of fabrics under varying external forces in dynamic scenes. Then we study what visual cues allow humans to achieve this perceptual constancy. The stimuli are animated videos containing a hanging fabric moving under oscillating wind. We vary both intrinsic mechanical properties such as mass and stiffness of the cloth as well as the strength of the wind force. We discuss our results in the context of optical flow statistics. This advances the current understanding of the role of motion in perception of material properties in dynamic scenes.

Keywords: Perception, Cloth Simulation, Image Processing Concepts: •Computing methodologies $\rightarrow$ Graphics systems and interfaces; Perception;

\footnotetext{
*e-mail:wb1918a@american.edu

Permission to make digital or hard copies of part or all of this work for personal or classroom use is granted without fee provided that copies are not made or distributed for profit or commercial advantage and that copies bear this notice and the full citation on the first page. Copyrights for thirdparty components of this work must be honored. Abstracting with credit is
}

\section{Introduction}

Material perception is useful because it helps us predict how to interact with objects in daily tasks. Previous research in human material perception has mainly focused on optical properties of rigid materials. Many materials in our environment, however, are soft and deformable and humans are good at perceiving their mechanical properties (e.g. stiffness) as well as their optical properties (e.g. surface gloss) [Adelson 2001; Sharan et al. 2014]. Accurately estimating mechanical properties is especially important for action planning because they help us predict what type of action can be done upon the object. Hence, it is important in robotics and virtual reality technologies [Song et al. 2005]. Nonetheless, little is known about how humans perceive mechanical properties.

Previous research suggests that motion can be used as a cue for estimating mechanical properties of cloth [Bouman et al. 2013]. Estimating mechanical properties in a dynamic scene is difficult because both the intrinsic properties of the fabric and the external force contribute to the apparent motion of the cloth. For example, it might be difficult for humans to distinguish a piece of heavy cloth under strong wind force from a piece of light cloth under weak wind (see Figure 1). Material perception in dynamic scenes is only useful when it is invariant across external forces. In real life, it is impossible to have constant external forces across different scenes. In order to achieve invariant material perception, humans must discount the effects of the wind force and extract the intrinsic mechanical properties of the cloth.

permitted. To copy otherwise, or republish, to post on servers or to redistribute to lists, requires prior specific permission and/or fee. Request distribute to lists, requires prior specific per
permissions from permissions@acm.org

Copyright held by the owner/author(s).Publication rights licensed to ACM. SAP '16, July 22-23, 2016, Anaheim, CA, USA

ISBN: 978-1-4503-4383-1/16/07 ...\$15.00

DOI: http://dx.doi.org/10.1145/2931002.2931016 
This line of questioning leads to the following question: what kind of motion cues are diagnostic of the intrinsic mechanical properties of materials, despite variation of external force? Physics-based cloth simulation provides us a forward model describing how physical parameters together with scene parameters contribute to apparent motion of cloth as well as ways to control both appearance and dynamics systematically. In this paper, we use physics-based cloth animations as our stimuli and vary both the intrinsic mechanical properties and the strength of the external wind force. In order to focus on the effect of motion, we keep the appearance of the cloth constant. We measure how external wind force affects perceptual estimation of cloth mechanical properties: bending stiffness and mass. Can observers infer the physical parameters and make invariant judgement of mechanical properties when the external force is different? Can we explain our results with analyzing the statistics of optical flow fields of the videos?

The contributions of the paper are, first, we investigated how humans estimate mechanical properties of fabrics in dynamic scenes, which was previous ignored, and measured whether humans can achieve invariant estimation of these mechanical properties under variation of external force. Second, we analyzed optical flow statics of the videos and identified the statistics that best correlated with the perceptual results. Our results furthered the understanding of the role of motion in human material perception and will be useful for developing video editing softwares that manipulate image statistics to modify the perceived mechanical properties of cloth.

\section{Related Work}

Recent advances in cloth simulations allow high fidelity modeling of photo-realistic appearance that mimic the behavior of real cloth. Many models has been developed to describe the dynamic behavior of cloth. Due to the limited space, we refer the reader to [Miguel et al. 2013; Narain et al. 2012; Hauser et al. 2003] for reviews of recent cloth models. In this paper, we use Blender cloth solver to describe how a piece of cloth is moved by wind force.

\subsection{Perception of cloth dynamics}

There has been many works on understanding perception of material properties, but most have focused on optical properties (e.g. surface gloss and translucency) of solid objects (for a review, see [Fleming 2014]). Little is known regarding perception of deformable objects in dynamic scenes. Bouman et al. 2013 [2013] performed the first perceptual study of humans'ability to estimate the mechanical properties of real fabric from images and videos. They find that human perception from videos correlated well with the ground truth measurements of stiffness and mass. In their study, humans only estimated the cloth material properties when the wind force is kept the same between the compared conditions. In our paper, we investigated whether humans can extract invariant mechanical properties when the wind strength was varied within the compared fabrics. In addition, since they used the real cloth, it is impossible to know how appearance affects the perceived mechanical properties. It is possible that observers can tell one fabric is stiffer than another simply by looking at the appearance (e.g. textures, glossiness,) without using the dynamic information. In fact, recent work suggested that appearance has a dominant effect in comparison to dynamics on cloth perception [Aliaga et al. 2015] and perception of shape deformation of deformable objects [Han and Keyser 2015]. In this paper, we kept the appearance constant across our stimuli and measure how well observers can extract physical parameters describing the mechanical properties and show dynamics alone can be used to estimate mechanical parameters in variation of external wind force.

McDonnell et al. [2006] performed perceptual experiments to validate the use of level-of-detail (LOD) models for cloth simulation.
They evaluated the impact of impostors and low resolution geometries on displaying material properties (bending stiffness) of animated cloth and show that impostors are very effective at depicting the deformation properties of clothing. Sigal et al. [2015] developed a perceptual control space for simulation of cloth on virtual characters that works with any physical simulator. Their algorithm learned from a set of perceptual rating experiments to map large number of parameters from a physical simulator to a few intuitive perceptual parameters of cloth attributes. Their measurements focused on the perception of high-level cloth attributes and didn't investigate how humans estimate mechanical properties under wellcontrolled external force. Aliaga et al.[2015] studied the interplay between dynamics and visual appearance on cloth perception using videos of real cloth and animations. They found that, in most of the cases, appearance dominated over dynamics, except for the special case of silk, where the dynamics is very characteristic of the fabric. In our study, we keep the appearance constant across the dynamic scenes and focus on how observers estimate mechanical properties of cloth from dynamics alone. In addition, [Aliaga et al. 2015] used a matching task to ask observers to rate overall similarity between two pieces of fabrics across different videos (with a combination of different appearance/dynamics). Here, we specifically ask observers to rate a mechanical property, stiffness or mass, from a dynamic scene using a paired-comparison task.

\subsection{Motion and material perception}

Motion has been proposed to play a role in material perception [Marlow and Anderson 2016]. Doerschner et al. [2011] identified three motion cues that can be used to distinguish matte and shinny materials. They show that these motion measurements can override static cues, leading to dramatic changes in perceived material depending on the image motion characteristics. Besides cloth, [Kawabe et al. 2015] et.al. proposed that optical flow statistics can be used to estimate liquid viscosity by showing that observers can judge liquid viscosity using stimuli constructed by noise fields extracted from the optical flow of the original video. They further showed how optical flow statistics are correlated with impression of liquid in these videos. Inspired by this work, we investigate how optical flow statistics are correlated with perceived bending stiffness and mass of fabrics in videos.

\section{Experiment}

The goal of the experiment is to investigate whether people can estimate the intrinsic material properties of fabrics under variation of external force. We choose a simple dynamic scene containing a piece of fabric moved by oscillating wind force. We vary both the intrinsic mechanical properties (bending stiffness and mass) as well as the strength of the external wind force and measure how well humans can achieve invariant perception of mechanical properties of materials.

\subsection{Stimuli}

The cloth dynamics were simulated using Blender cloth engine (version 2.67). Cloth was modeled as a triangular mesh and a massspring model was used to define how the piece of cloth interacts with external force [Provot 1995]. The cloth-object collision was described using the algorithm proposed by Mezger et al. [2002]. There are mainly three parameters describing the intrinsic mechanical properties of cloth: bending stiffness, mass, and structural stiffness. Bending stiffness descries how the cloth forms its wrinkles. Higher values result in bigger but not necessarily more wrinkles. Mass describes the heaviness of cloth per area. Structural stiffness describes the elasticity of the cloth, with lower values making the cloth more rubber-like. We also kept the parameters spring damp, air damp, and friction (which has little effect in our scene) constant across the videos. In a previous study using multi-dimensional scal- 
Which piece of fabric looks stiffer?

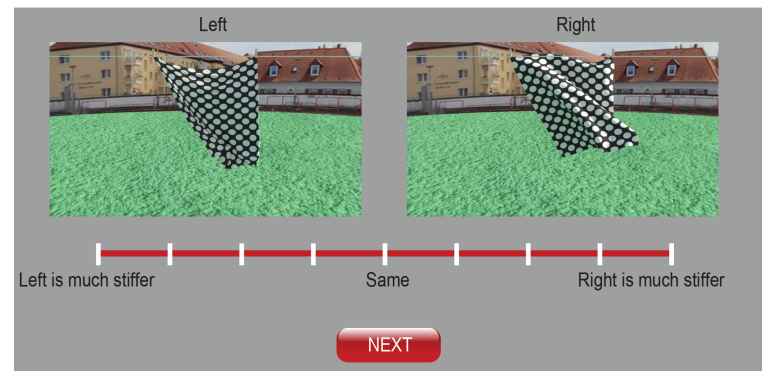

Figure 2: Experimental setup of pairwise comparisons of material properties from video stimuli. Observers are asked to compare bending stiffness of the two fabrics on a 9 point scale. A similar user interface is used in the mass rating experiment.

ing,[Xiao and Kistler 2015] discovered that structural stiffness has minimal effects on perception of material properties in a similar scene. In this study, we only varied mass and bending stiffness. We used a polka-dot texture to render the appearance of all the fabric across the videos. We believe the polka-dot texture don't bias toward any particular categories of fabrics. The scene was lit by an area light (Blender's sun lamp), which was located at the upper right corner in front of the fabric. For each video, a wind source was randomly set to be either at the left or the right side of the fabric. The wind was oscillating in the horizontal plane perpendicular to the fabric and the angle is ranging from 60 to 90 degrees. The resulting video was 10 seconds in length with 24 frames per seconds, which was rendered using Blender's cycles renderer.

\subsection{Task}

A paired-comparison method was used to measure perceived differences in the bending stiffness and mass between the fabrics in two videos (see Figure 2). In the bending stiffness task, each observer was shown two videos of fabric stimuli moving by either the same or a different wind force and then was asked to report which fabric, left or right, is stiffer by answering on a 9-point scale provided underneath the videos. Figure 2 shows the experiment interface. This pairwise score, which takes a value in $\{-4,-3,-2,-1,0,1,2,3,4\}$, indicates which fabric the observer believed is stiffer, and the degree of stiffness difference between two fabrics. Similarly, in the mass task, an observer was asked to report a pairwise score indicating the relative weight of the fabric. Since all the fabrics have the same size in the videos, this will be the same as mass. Stimuli were presented on a LCD monitor (Dell 27 Ultra HD $3840 \times 2160$ resolution) on each trial with $60 \mathrm{~cm}$ distance from the observer. Before the experiment, the observer is familiarized with the concept of bending stiffness and mass by watching videos of real fabrics. Observers were told that the wind strength might or might not be the same across the videos.

In bending stiffness group, we used 7 different bending stiffness values $(0.05,0.25,0.5,5,10,80,150)$ and kept the mass values constant at 0.5 . For mass group, we used 7 different mass values $(0.15$, $0.2,0.3,0.5,0.7,1,1.3)$ and kept the bending stiffness constant at 5 . We used four wind strengths $(250,350,450,550)$. The values of bending stiffness and mass were sampled based on the values of the preset materials in Blender ( silk $(0.05,0.15)$, cotton $(0.5,0.3)$, denim(10,1), and leather $(150,3))$. This together resulted in a total of 28 videos for each experiment and 378 pair-comparisons. Seven observers ( 6 females, mean age 22.8 years) finished the bending stiffness task and another seven ( 5 females, mean age 22 years) finished the mass task. All the observers have normal visual acuity and color vision.
A.
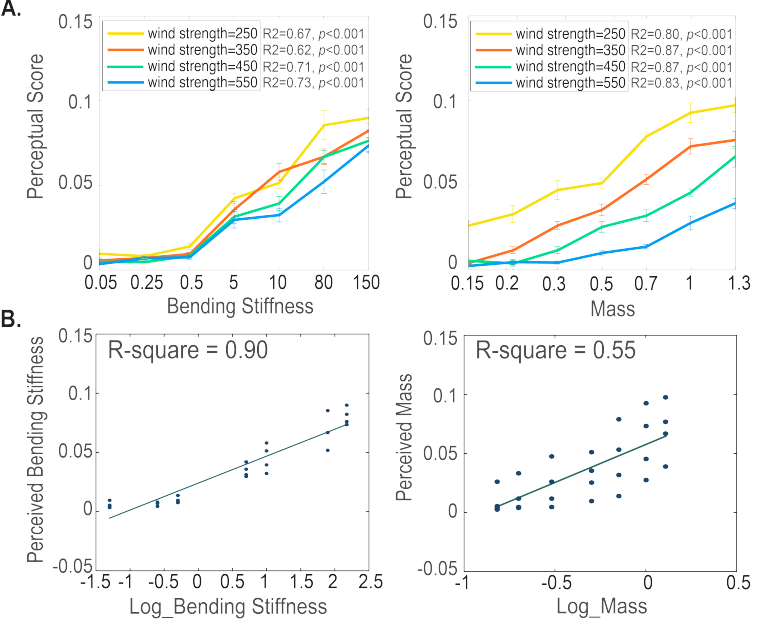

Figure 3: Perceptual results of the bending stiffness task (left panel of Figure 3A) and the mass task (right panel of Figure 3A). The figures plot averaged perceptual score over physical parameters under 4 wind strengths. Perceptual score is significantly correlated with bending stiffness and mass under each of the four wind strengths. The correlations still held when all wind strengths were collapsed. Figure $3 B$ plotted the perceptual score as a function of log bending stiffness (left panel) and log mass (right panel). Error bars are SEM across the observers.

\section{Results and Discussion}

\subsection{Perceptual Results}

The pair-wise ratings of 378 trials for each observer are converted to perceptual scores using the Bradley-Terry model [Caron and Doucet 2012]. Figure 3A shows the relationship between the mean perceptual score and the physical parameters (bending stiffness and mass, respectively). As the figure shows, across all the wind conditions, perceptual scores of stiffness and mass are both positively correlated with the physical parameters $\left(R^{2}=0.73, \mathrm{p}<.001\right.$, MSE $=$ $0.017, \eta^{2}=0.74$ for bending stiffness and $R^{2}=0.75, \mathrm{p}<.001, \mathrm{MSE}=$ $0.013, \eta^{2}=0.57$ for mass). When plotted against log physical parameter (Figure $3 \mathrm{C}$ ), the perceptual score could be well fitted by a linear function ( $R=0.90$ for bending stiffness and $R=0.55$ for mass). This indicates that observers are able to estimate the mechanical properties of the fabrics in the dynamic scene.

We further analyze the effect of wind by a one-way ANOVA with wind strength as the factor. Results show that wind strength has a main effect on perceived mass $(\mathrm{F}(3,24)=4.64, \mathrm{p}<0.01, \mathrm{MSE}=0.003$, $\eta^{2}=0.36$ ). A post-hoc Tukey test shows that fabrics under the 250 wind strength are rated significantly heavier than the fabrics under the 550 wind strength $(\mathrm{p}<0.05)$. In contrast, the wind strength doesn't have a significant effect on perceived bending stiffness $\left(\mathrm{F}(3,24)=0.257, \mathrm{p}=0.86, \mathrm{MSE}=0.000, \eta^{2}=0.04\right)$, indicating that observers could discount the effect of wind and show a perceptual constancy of bending stiffness.

\subsection{Computational Analysis}

In order to find out what motion cues observers used to estimate the mechanical properties in variation of the wind force, we computed the optical flow fields for two consecutive frames for each of the videos, using the method described in [Sun et al. 2014]. Figure 4A shows examples of video frames from the experimental conditions and their optical flow fields. First, the examples show that the optical flow fields of the stiffer material have more shifted pixels 


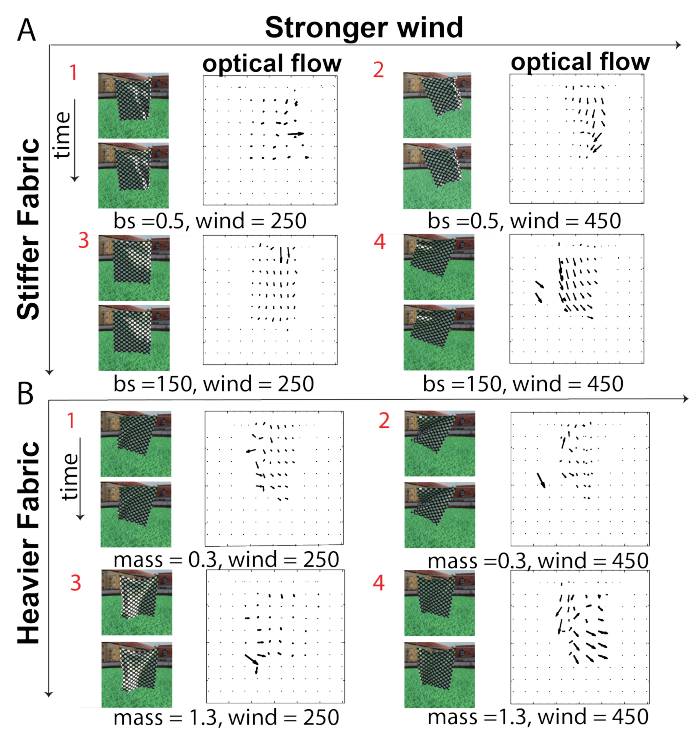

Figure 4: Examples of optical flow fields of videos of various materials and wind strength conditions. Panel A shows the results for videos used in the bending stiffness task and Panel B shows the results for the videos used in the mass task. The images are two consecutive video frames from the corresponding stimuli.

(the vectors with non zero magnitudes) than that of the less stiff material (Figure 4A1 and 4A3, Figure 4A2 and 4A4). Specifically, larger vectors are concentrated along the edges in the flow field of the less stiff material (Figure 4A1 and 4A2) while larger vectors are more distributed across the whole flow field of the stiffer material. Second, as the wind becomes stronger, the plot shows that there are wider distributions of the magnitudes of the motion vectors. This effect is particularly strong for the stiffer materials (Figure 4A3 and 4A4). Moreover, The stiff fabric under the strong wind has very different patterns as the less stiff fabric under the weak wind (Figure $4 \mathrm{~A} 1$ and $4 \mathrm{~A} 4)$

To understand which features of the optical flow can be used to predict our perceptual results, we examine the contribution of 16 motion statistics to the perceptual score of bending stiffness, as well as the physical bending stiffness, and the wind strength using linear regression (see the left panel of Figure 5). The details of the calculation of these statistics is described in [Kawabe et al. 2015]. The figure shows different optical flow statistics are affected by wind strength and the physical parameters. Specifically, Laplacian skewness is sensitive to wind strength, while speed mean is sensitive to both physical parameters and perceptual scores. This can explain our perceptual results that the wind strength has little effect on perceived bending stiffness, possibly because the observers are not sensitive to the information conveyed by the change in the wind strength to infer bending stiffness.

Figure 4B shows examples of optical flow fields of videos used in the mass rating experiment. Unlike the bending stiffness, it is difficult to disassociate the effects of wind and physical mass parameters on the optical flow fields. In other words, the effects of physical parameters on optical flow patterns depend on the wind conditions. The lighter fabric under the weak wind has a similar pattern as the heavier fabric under the strong wind (Figure 4B1 and B4). That might be the reason why observers cannot discount the effects of wind. We conjecture that instead of using only motions cues, observers can use simple shape cues in the videos to estimate the mass of the fabric (e.g. light fabric can be blown to an upward position and heavier fabric stays lower).

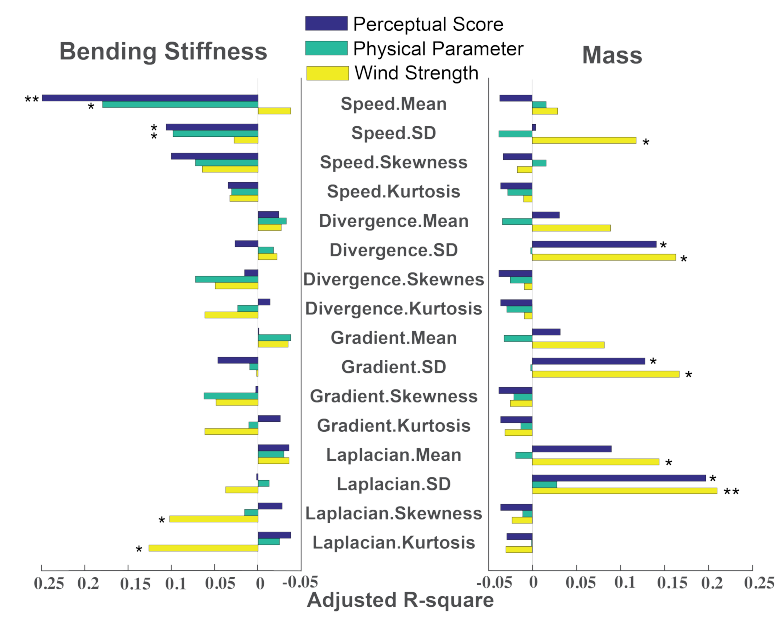

Figure 5: Adjusted $R^{2}$ in the single regression analysis of perceptual score (blue), physical parameters (green), and the wind strength (yellow) as a function of each optical flow statistics. The * denotes $p<0.05$, ** denotes $p<0.01$.

The right panel of Figure 5 shows that perceptual scores are sensitive to standard deviations of divergence, gradient, and Laplacian, which are also sensitive to wind strength. This can explain our perceptual results that the wind strength has a significant effect on perceived mass.

\subsection{Limitations and future work}

In this paper, we used Blender to simulate cloth dynamics. Even though Blender doesn't simulate cloth dynamics with perfect physical accuracy, our observers reported experiencing a vivid impression of cloth and can distinguish fabrics with different mechanical properties. This implies that the simulated cloth shares essential visual cues from the real cloth. Therefore, we believe our findings can be generalized to other cloth simulations. In the future, we will explore other more sophisticated approach of cloth simulations and study the effects of different types of dynamic scenes on cloth perception. Past studies show that for certain fabrics, textures play dominant roles in perceiving cloth properties [Aliaga et al. 2015].This study focused on the effects of dynamics on perceived mechanical properties by using the same texture across experimental stimuli. We plan to systematically manipulate textures including optical properties such as surface gloss and measure the perception of mechanical properties.

\section{Conclusion}

In this paper, by combining psychophysics, computer graphics, and image processing, we show how humans estimate mechanical properties of fabrics from dynamics scenes. We vary both the strength of the wind force as well as bending stiffness and mass of the fabrics from trial to trial. We find perceptual estimation of the mechanical properties are highly correlated with the physical parameters used in the cloth model, indicating observers can distinguish material properties based on dynamics information. The data also suggests that the external wind strength has little effect on perceived bending stiffness but has a significant effect on perceived mass. We show that the patterns of the optical flow extracted from the videos can be diagnostic of intrinsic bending stiffness independent of wind condition. Further computation of statistics of optical flow reveals that the speed mean of the flow fields is correlated with perceived bending stiffness as well as the physical parameters. Our results demonstrate how the visual system exploits motion statistics for estimating mechanical properties of cloth in dynamic scenes. 


\section{Acknowledgements}

The authors wish to thank Shaiyan Keshvari for editing the manuscript. The research is partially funded by the faculty startup funding and faculty research support grant by American University (Bei Xiao). The authors also wish to thank the high performance computing system provided by American University for rendering and computation support.

\section{References}

ADELSON, E. H. 2001. On seeing stuff: the perception of materials by humans and machines. In Photonics West 2001-electronic imaging, International Society for Optics and Photonics, 1-12.

Aliaga, C., O’Sullivan, C., Gutierrez, D., And TaMSTORF, R. 2015. Sackcloth or silk?: the impact of appearance vs dynamics on the perception of animated cloth. In Proceedings of the ACM SIGGRAPH Symposium on Applied Perception, ACM, 41-46.

BARAFF, D., AND WITKIN, A. 1998. Large steps in cloth simulation. In Proceedings of the 25th annual conference on Computer graphics and interactive techniques, ACM, 43-54.

Bouman, K., Xiao, B., Battaglia, P., and Freeman, W. 2013. Estimating the material properties of fabric from video. In Proceedings of the IEEE International Conference on Computer Vision, 1984-1991.

CARon, F., AND Doucet, A. 2012. Efficient bayesian inference for generalized bradley-terry models. Journal of Computational and Graphical Statistics 21, 1, 174-196.

CHOI, K.-J., AND Ko, H.-S. 2005. Stable but responsive cloth. In ACM SIGGRAPH 2005 Courses, ACM, 1.

Davis, A., Bouman, K. L., Chen, J. G., Rubinstein, M., DURAND, F., AND FREEMAN, W. T. 2015. Visual vibrometry: Estimating material properties from small motions in video. In Computer Vision and Pattern Recognition (CVPR), 2015 IEEE Conference on, IEEE, 5335-5343.

Doerschner, K., Fleming, R. W., Yilmaz, O., Schrater, P. R., HARTUnG, B., AND Kersten, D. 2011. Visual motion and the perception of surface material. Current Biology 21, 23, 2010-2016.

FLEMING, R. W. 2014. Visual perception of materials and their properties. Vision research 94, 62-75.

Han, D., AND Keyser, J. 2015. Effect of appearance on perception of deformation. In Proceedings of the 14th ACM SIGGRAPH / Eurographics Symposium on Computer Animation, ACM, New York, NY, USA, SCA '15, 37-44.

HAuser, K. K., SHen, C., AND O’BRIEN, J. F. 2003. Interactive deformation using modal analysis with constraints. In Graphics Interface, vol. 3, 16-17.

KaWabe, T., Maruya, K., Fleming, R. W., and Nishida, S. 2015. Seeing liquids from visual motion. Vision research 109 , 125-138.

MARlow, P. J., AND Anderson, B. L. 2016. Motion and texture shape cues modulate perceived material properties. Journal of vision 16, 1, 5-5.

McDonnell, R., DobByn, S., Collins, S., AND O'Sullivan, C. 2006. Perceptual evaluation of lod clothing for virtual humans. In Proceedings of the 2006 ACM
SIGGRAPH/Eurographics symposium on Computer animation, Eurographics Association, 117-126.

Mezger, J., Kimmerle, S., And Etzmuss, O. 2002. Progress in collision detection and response techniques for cloth animation. In Computer Graphics and Applications, 2002. Proceedings. 10th Pacific Conference on, IEEE, 444-445.

Miguel, E., Bradley, D., Thomaszewski, B., Bickel, B., Matusik, W., OtaduY, M. A., And Marschner, S. 2012. Data-driven estimation of cloth simulation models. In Computer Graphics Forum, vol. 31, Wiley Online Library, 519-528.

Miguel, E., Tamstorf, R., Bradley, D., Schvartzman, S. C., Thomaszewsi, B., Bickel, B., Matusik, W. MARSChNer, S., AND OtAdUY, M. A. 2013. Modeling and estimation of internal friction in cloth. ACM Transactions on Graphics (TOG) 32, 6, 212.

Narain, R., SAmit, A., And O'Brien, J. F. 2012. Adaptive anisotropic remeshing for cloth simulation. ACM Transactions on Graphics (TOG) 31, 6, 152.

Provot, X. 1995. Deformation constraints in a mass-spring model to describe rigid cloth behaviour. In Graphics interface, Canadian Information Processing Society, 147-147.

Sharan, L., Rosenholtz, R., And Adelson, E. H. 2014. Accuracy and speed of material categorization in real-world images. Journal of vision 14, 9, 12-12.

Sigal, L., Mahler, M., Diaz, S., Mcintosh, K., Carter, E., Richards, T., AND HodGins, J. 2015. A perceptual control space for garment simulation. ACM Transactions on Graphics (TOG) 34, 4, 117.

Song, A., Morris, D., Colgate, J. E., And Peshrin, M. A. 2005. Real time stiffness display interface device for perception of virtual soft object. In Intelligent Robots and Systems, 2005.(IROS 2005). 2005 IEEE/RSJ International Conference on, IEEE, 139-143.

Sun, D., Roth, S., AND Black, M. J. 2014. A quantitative analysis of current practices in optical flow estimation and the principles behind them. International Journal of Computer Vision 106, 2, 115-137.

XiaO, B., AND Kistler, W. 2015. Perceptual dimensions of material properties of fabrics in dynamic scenes. Journal of vision $15,12,938-938$. 